\title{
DYNAMICAL EVOLUTION OF BINARY CLUSTERS AND COLLIDING GALAXIES
}

\author{
D. SUGIMOTO, T. EBISUZAKI, J. MAKINO \\ Department of Earth Science and Astronomy \\ College of Arts and Sciences \\ University of Tokyo \\ 3-8-1 Komaba, Meguro, Tokyo 153 \\ Japan
}

\begin{abstract}
Merging of stellar systems is investigated by means of $\mathrm{N}$-body simulations. We discuss the synchronization instability to trigger sudden merging, the origin of the ellipticity in the merger remnants, the distribution of stars in the halo, and the survival of the colour gradient of stars against the violent relaxation of the merging process.
\end{abstract}

\section{Physics of merging process}

In the context of N-body simulation, merging of binary globular clusters and of colliding spherical galaxies are the same problem; the difference lies only in their initial orbital parameters, whether initially in bound orbit or not. We summarize physical understanding of the merging processes.

The merging process of a binary globular cluster consisting of equal-mass components was discussed by Sugimoto \& Makino (1989). Here the tidal force between component clusters quickly circularizes the orbit and in the inertial frame the potential field rotates, and stars in the halo of the cluster are accelerated to gain energy and angular momentum. As such stars escape, the orbital angular momentum reduces and the separation between the components shrinks, resulting in increased orbital angular velocity. The spins of the components synchronize to it at the expense of the orbital angular momentum. This makes the separation still smaller and the spin angular velocity still higher. This feed-back becomes divergent when the separation reaches a critical value and is called synchronization instability. Since this instability begins at a critical separation, the resultant mergers have almost unique structure, irrespective of the initial orbital parameters. For example, the figure of the merger is an oblate spheroid with ellipticity of about 0.3 as observed in the youngest globular clusters in the Magellanic Clouds (MCs).

Similar simulation has been done by Makino et al. (1990) for a pair of globular clusters whose initial mass ratio was $2: 1$. The radius ratio was taken as $1: 1$. A phase of mass transfer is found to precede the synchronization instability with about $20 \%$ of particles of the secondary (less massive) component overflowing its Roche lobe to accrete on the primary. By comparing the figure of the merger remnant of the unequal-mass case with that of the equal-mass case, we find that their ellipticities are almost the same though the specific angular momentum (i.e. per unit mass) of the merger is only $60 \%$ of the equal-mass case. This implies that an appreciable part of the ellipticity is to be ascribed not only to the rotation but also to the anisotropy of the velocity dispersions, i.e. the difference of velocity dispersions in the initial orbital plane and perpendicular to it. 
The radial distribution of the number density $\rho$ of stars in the halo of the merger is well represented as $\rho \sim \mathrm{r}^{-4}$. Makino et al. (1990) have shown that such distribution is merely the result of a relation between the energy space and the configuration space. A wide radial range in the halo region corresponds only to a small range near $E=0 \_$. Therefore, the gradient of the distribution function in the energy space hardly affects the distribution function in $r$ space. When such distribution is seen projected on the sky, it is consistent with de Vaucouleur's $r^{1 / 4}$ law. The only necessary condition for such distribution is that the system has suffered from a violent relaxation to produce a number of particles near $E=0 \_$. Such a distribution is found in globular clusters of the MCs and in some giant elliptical galaxies and is not inconsistent with the merger hypothesis for their origin.

Secular evolution of the merger remnant is calculated by Akiyama et al. (in prep.); the remnant becomes rounder with the time scale of the gravothermal catastrophe. There are two effects: a decrease of the anisotropy in the velocity dispersions; and the outward transport of angular momentum along the radial gradient of angular velocity produced by gravothermal contraction.

\section{Survival of colour gradient against merging}

Head-on collision of galaxies is another extreme problem and is simulated with our special purpose computer GRAPE-1 (Sugimoto et al. 1990) by representing each galaxy with 8192 mass points.

The initial orbital energy is set vanishing. For two galaxies to merge the collision should be inelastic. This is realized by ejecting some mass points from the system. Since the amount of mass ejection is not abundant enough during one passage, an oscillation takes place. Afterwards, the two galaxies merge. Notice that the colour gradient survives well despite such violent relaxation. A similar result is also seen in the merging of binary globular clusters. As for the merger hypothesis for the origin of giant ellipticals, the fear was that the violent relaxation shuffled the initial distribution of stars, destroying the initial colour gradient in the parent galaxies. Our simulation shows that it is so. This implies that the violent relaxation is not violent in certain respects.

Ichikawa (1990) showed analytically that phase mixing proceeds at different speeds in different directions. In particular, when a certain amount of mixing has been done within equi-energy hypersurfaces, mixing in the direction orthogonal to the equi-energy hypersurfaces becomes suppressed. This implies that after a certain mixing diffusion in the energy space proceeds only with a power law in time, though diffusion in other directions proceeds exponentially. This is because the energy of a mass point changes only through the fluctuation of the mean field (within the collision-free regime), but the fluctuation itself almost ceasees when mixing in the other directions has proceeded a certain amount. Since the energy bins are approximately translated to the bins in the radial distance, the colour gradient survives well also in the radial direction.

Another point worth mentioning, is that the merger remnant is a prolate spheroid with the longest axis parallel to the initial separation. The shape and the orientation of the axis of rotation will give us information about the orbital energy and impact parameter in the initial stellar system.

\section{References}

Sugimoto, D., Makino, J. (1989), Publ. Astron. Soc. Japan 41,1117.

Makino, J., Akiyama, ?, Sugimoto, D. (1990), in press.

Sugimoto, D., Ebisuzaki, T., Ito, T., Makino, J. (1990), this Symposium.

Ichikawa, ?, (1990), MSc thesis, University of Tokyo. 